\title{
3D VELOCITY MODEL BUILDING - AN ACCURATE AND EFFICIENT INTERPRETIVE WORKFLOW
}

\author{
MURRAY ROTH', DAVID KESSLER' ${ }^{1}$ and DAVID M. SIBLEY ${ }^{2}$ \\ 'Landmark Graphics Corp., 15150 Memorial Drive, Houston TX 77079, USA \\ ${ }^{2}$ Chevron USA Productions Company
}

\section{Introduction}

The workflow for creating accurate 3D depth images varies widely in cost and effort, depending largely on data complexity. Much of the effort associated with depth imaging is associated with the construction of an accurate macro velocity model. Depth imaging is an integral component of velocity model building, providing both velocity and depth horizon information. Both the cost and effort of performing 3D depth imaging and, hence, velocity model building increases as data complexity rises from simple to moderate and on to complex geology. In this paper, we will describe an accurate and efficient interpretive workflow for $3 \mathrm{D}$ velocity model building for data with simple-to-moderate levels of geologic complexity.

\section{Velocity Model Building}

In order to perform 3D depth imaging, an accurate macro interval velocity model must be constructed. This velocity model is comprised of velocity and depth information, which may be derived from various sources, depending upon data availability and geologic complexity.

If available, a priori, in situ velocity/depth information, such as sonic logs, check shots and VSP data are extremely valuable for velocity model construction and verification. In regions of simple geology, ray-based tools are commonly used for velocity estimation (e.g. coherency inversion) (Landa et al., 1988) and depth horizon determination (e.g. map migration) (Sorin et al., 1993, Sattlegger, 1985). Moving into regions of moderate geologic complexity, poststack wave-equation techniques (e.g. poststack depth migration) (Ratcliff et al., 1994) are commonly used to facilitate depth horizon estimation. Finally, in regions of complex geology, pre-stack wave equation techniques (e.g. constant-velocity-half-space) (Reshef, 1994) are used to estimate velocities and depth horizon positions.

\section{The Workflow}

The workflow examined in this study uses a variety of interpretive techniques and tools to construct a macro velocity model. Our focus in this paper is upon imaging stacked 3D datasets, with simple to moderate geologic complexity. Consequently, we will rely upon integration of a priori information, including well log and synthetic data, for velocity assignment, while depth horizon information will be determined from the seismic data.

In regions of simpler geologic complexity, tools for automated horizon interpretation are invaluable in efficiently defining time horizon surfaces, on either unmigrated or time migrated 3D data volumes. Surface visualization and mapping applications are useful for horizon editing, smoothing and velocity cube construction. 
In regions of simpler geologic complexity, tools for automated horizon interpretation are invaluable in efficiently defining time horizon surfaces, on either unmigrated or time migrated 3D data volumes. Surface visualization and mapping applications are useful for horizon editing, smoothing and velocity cube construction.

Velocity information, assigned from well $\log$ information, can be used to map-migrate interpreted time horizons into depth, applying either a normal ray or image ray correction, as appropriate.

Editing, smoothing and quality analysis of the map-migrated depth horizon is efficiently performed using a mapping application, as illustrated in figure 1.

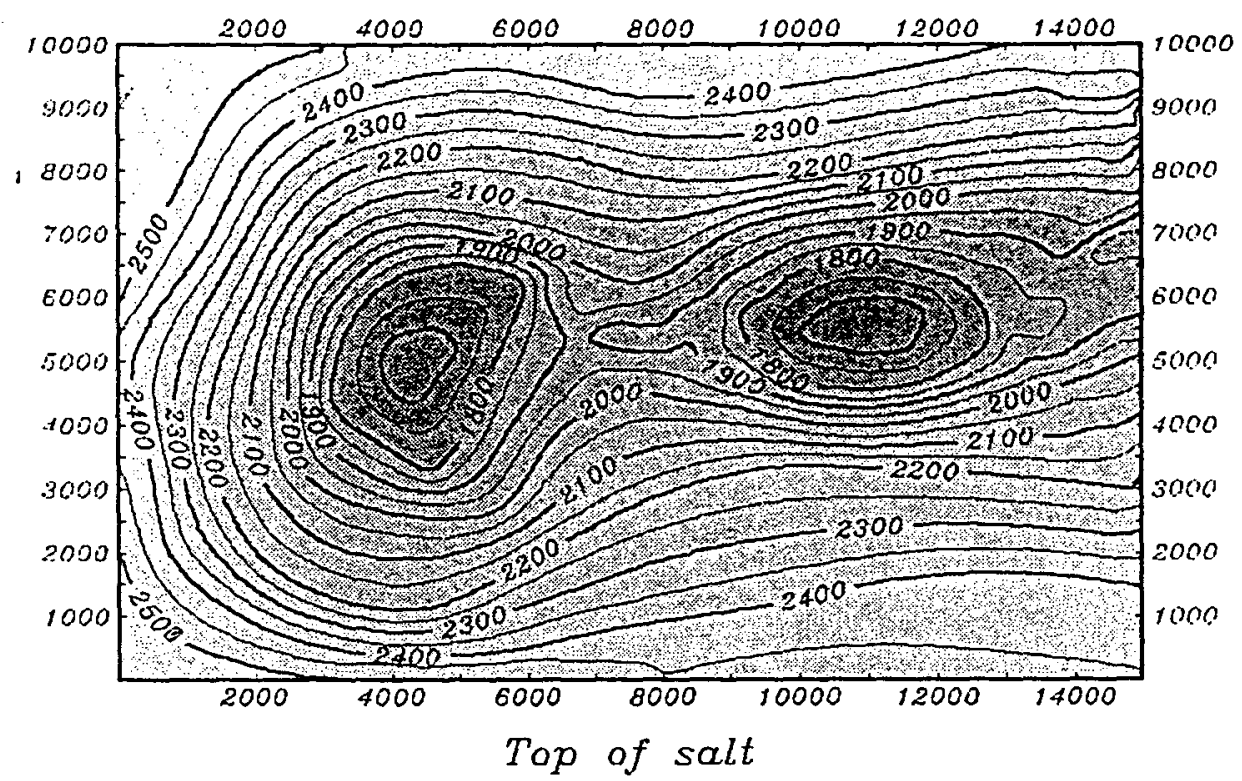

Figure 1: map view of migrated top-salt depth horizon

Edited depth horizons can be further analyzed, or integrated with velocity information using surface-visualization options, as illustrated in Figure 2.

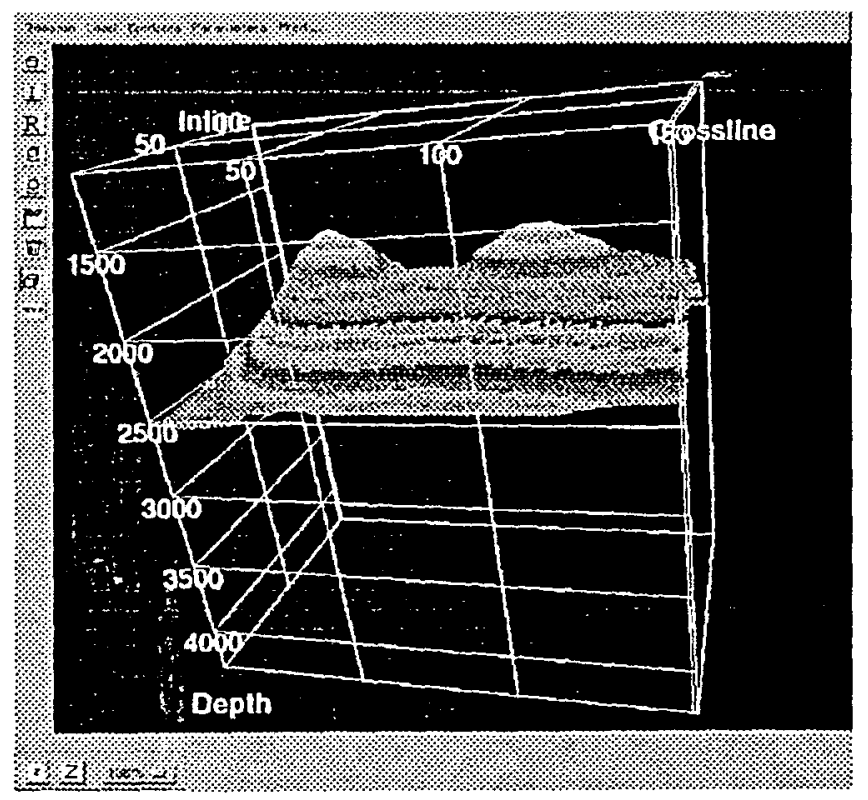

Figure 2: $3 D$ visualization of migrated top-salt depth horizon 
In regions of moderate geologic complexity, poststack 3D depth migration is used to facilitate depth horizon estimation. A priori salt velocity information is introduced to the velocity model as a constant-velocity-half-space, beneath the estimated top-of-salt surface. This velocity model is used to apply a 3D F-X-Y depth migration to the 3D seismic data volume. Once a 3D depth image is obtained, automated horizon tracking can be used to determine the base-of-salt surface from the seismic depth volume. Once again, mapping and visualization tools are an essential part of the workflow. A valuable integrated display is shown in figure 3 where the interpreted base-of-salt feature is show intersecting two vertical sections from the migrated 3D depth cube.

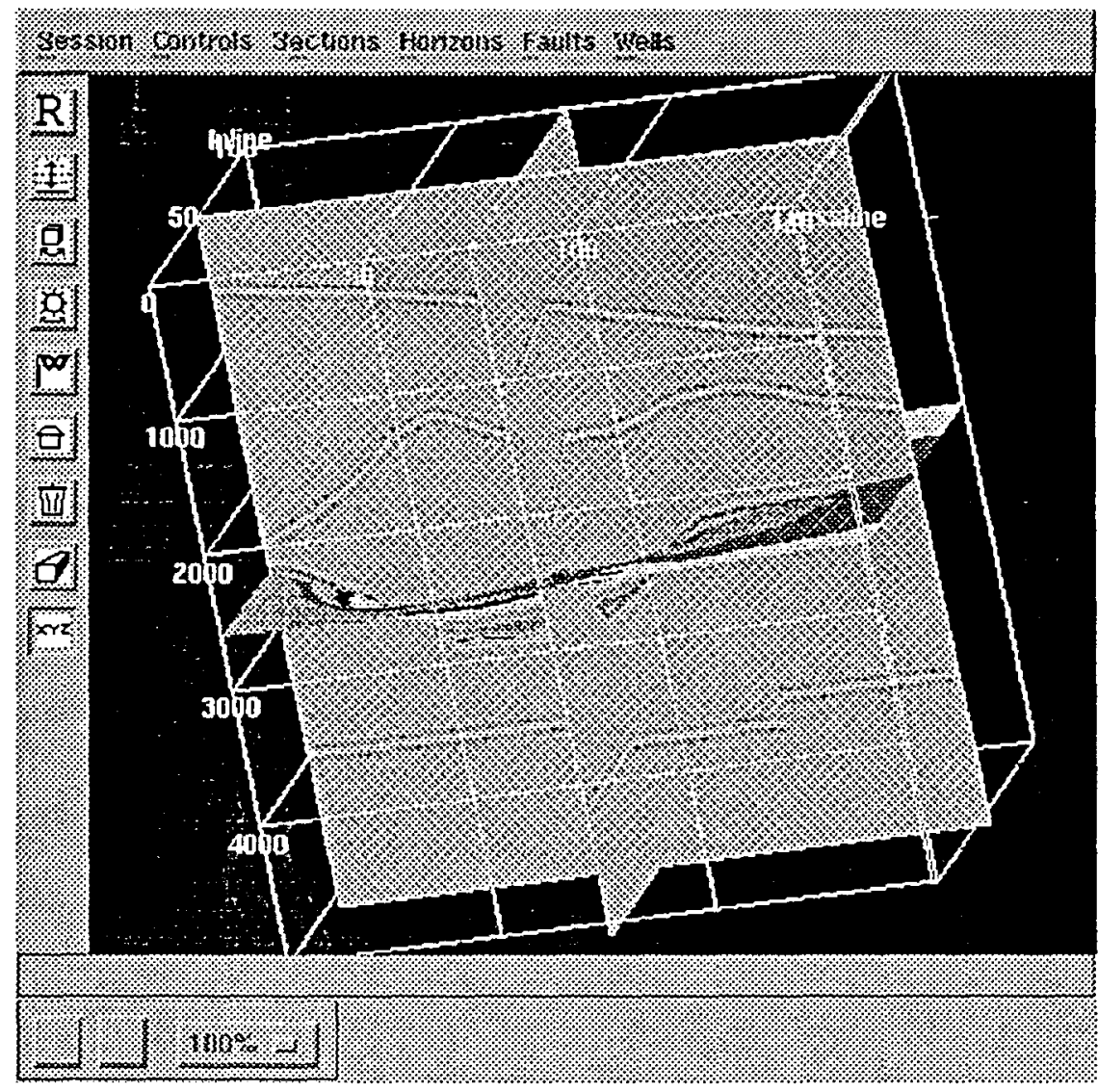

Figure 3: Poststack depth migration result is used to define the sub-salt layer

This iterative workflow is continued, using the appropriate tools and techniques, until a satisfactory $3 \mathrm{D}$ velocity depth model has been constructed. The surface visualization display in figure 4 illustrates the appearance of a velocity depth model comprised of 4 depth surfaces with velocity information shown as textured-mapped overlays.

\section{Practical Application of Workflow}

When applied in a production-oriented fashion, the interpretive workflow described herein can efficiently produce an accurate velocity depth model in a few iterative steps. A 3D marine dataset, exhibiting simple-to-moderately complex geology, is used to illustrate the practical application of this workflow. A series of images will be used to illustrate the iterative construction of a velocity depth model, using a variety of interpretive tools, well log information and 3D poststack seismic data. 


\section{Summary}

Increasingly, the need is growing for tools to facilitate the construction of $3 \mathrm{D}$ velocity depth models and corresponding depth-migrated seismic data volumes. Many exploration targets require prestack 3D depth imaging for velocity model building and accurate imaging. In many other regions, however, with simple-to-moderately complex geology, poststack ray and wave equation migration can facilitate accurate and efficient velocity depth model construction. An interpretive, iterative workflow has been described and illustrated, for building a velocity model with a priori well log information and 3D stacked seismic data, using a range of tools including: automated horizon interpretation, surface visualization, and sophisticated mapping tools. The efficiency and interpretive nature of this workflow makes it ideally suited for use in an integrated geoscience workstation environment.

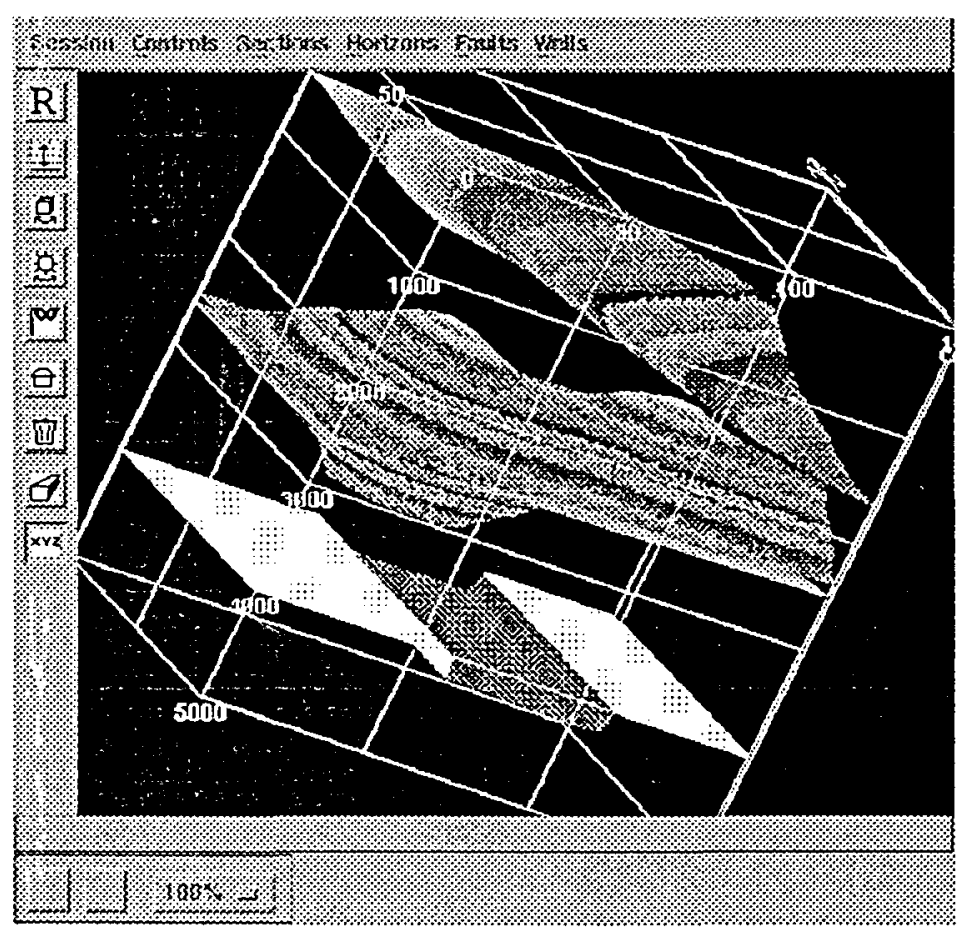

Figure 4: 3D surface visualization of the earth model

\section{References}

Landa E., Kosloff D., Keydar S., Koren Z. and Reshef M., 1988, A method for determination of velocity and depth from seismic data: Geophy. Prosp., vol. 36, p. 223-243.

Ratcliff D. W., Jacewitz C. A., and Gary S. H., 1994, Subsalt imaging via target-oriented 3D prestack depth migration: The leading edge, vol. 13, p. 163-170.

Reshef M., 1994, The use of 3D prestack depth imaging to estimate layer velocities and reflector positions: Joint EAEG/SEG summer workshop on construction of 3D macro velocity-depth models, the Netherlands.

Sattlegger J. W., 1985, Map migration and modeling algorithm: Expanded abstracts, 55th. SEG meeting, Washington D.C., p. 553-554

Sorin V., Keydar S., and Landa E., 1993, 3D kinematic inversion from a set of line profiles:

Geoph. Prosp., vol 41, p. 535-550. 